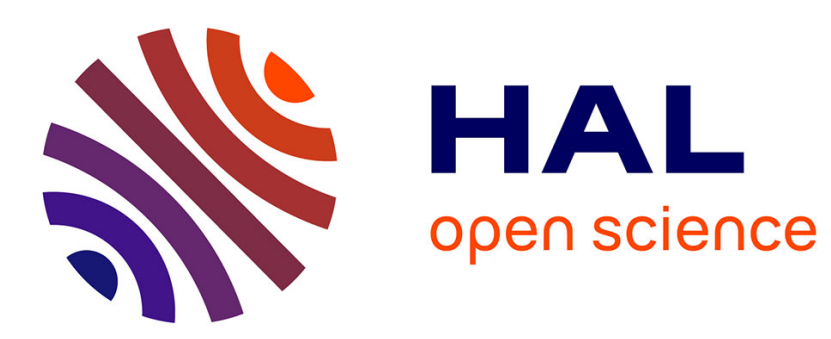

\title{
L'influence du vieillissement sur les pratiques touristiques en France
}

Pascal Pochet, Bernard Scheou

\section{To cite this version:}

Pascal Pochet, Bernard Scheou. L'influence du vieillissement sur les pratiques touristiques en France.

Espace Populations Sociétés, 2003, 2, pp. 303-315. halshs-00068913

\section{HAL Id: halshs-00068913 \\ https://shs.hal.science/halshs-00068913}

Submitted on 24 Jul 2006

HAL is a multi-disciplinary open access archive for the deposit and dissemination of scientific research documents, whether they are published or not. The documents may come from teaching and research institutions in France or abroad, or from public or private research centers.
L'archive ouverte pluridisciplinaire HAL, est destinée au dépôt et à la diffusion de documents scientifiques de niveau recherche, publiés ou non, émanant des établissements d'enseignement et de recherche français ou étrangers, des laboratoires publics ou privés. 


\section{L'influence du vieillissement sur les pratiques touristiques en France}

\section{Effects of the ageing of the population on tourism patterns in France}

\author{
Pascal Pochet \\ Chargé de recherche au \\ Laboratoire d'Economie des Transports \\ (Ecole Nationale des Travaux Publics de l'Etat, \\ Université Lumière Lyon 2, CNRS) \\ pochet@entpe.fr
}

\author{
Bernard Schéou \\ Maître de Conférence à l'Université de Perpignan, \\ chercheur associé au Laboratoire d'Economie des \\ Transports \\ scheou@entpe.fr
}

Résumé : Vieillissement de la population et changements de modes de vie à l'âge de la retraite, le secteur du tourisme est et sera fortement concerné par ces évolutions démographiques et sociales. Clientèle essentielle pour le secteur du tourisme, la catégorie des retraités et pré-retraités connaît de profonds changements, dont l'accroissement numérique ne constitue qu'un élément : élargissement de l'horizon de vie et amélioration de l'état de santé et des revenus, générations de plus en plus intégrées au monde de la consommation et des loisirs, mais générations qui seront aussi préoccupées par le financement des retraites et par les problèmes de mobilité aux grands âges. Comment ont évolué les pratiques touristiques des " seniors» ces dernière années, et comment risquent-elles de changer encore dans les années à venir? Nous présentons les évolutions récentes du tourisme des retraités à partir de données statistiques récentes, et essayons d'anticiper les évolutions futures. Sur bien des points les pratiques touristiques des personnes âgées se sont rapprochées de celles des plus jeunes. L'arrivée à la retraite des générations nées après-guerre va entraîner de nouvelles inflexions dans les pratiques touristiques, avec notamment une autonomie plus grande et plus de diversité dans les formes de voyages.

Mots-clefs : retraité, mode de vie, tourisme, demande, effet d'âge, effet de génération, enquête statistique
artAbstract : Ageing of the population, change in lifestyles of the elderly citizens, the tourism operators will be strongly affected by these long term socio-demographic evolutions. Retired persons already represent a large part of the clientele in the tourism sector. This group shows profound social change: growing life expectancy, improvement in health and standard of living, cohorts with an increasing participation in the consumption and leisure world, but also cohorts that will experience uncertainty as far as financing of retirement pensions is concerned, and that could face problems of mobility over 75 or 80 . What are the recent trends in matter of senior tourism, and how could it change during the next years? We present statistical data in order to highlight these topics. On much major points, tourism patterns of the elderly are getting closer to those of the younger adults, because of generation effects. The arrival at the retirement age of the baby-boomers cohorts will certainly imply new change in tourism demand of the elderly, especially a growing autonomy in tourism and travel, and a larger and larger diversity in the nature of holidays stays.

Key words : elderly people, lifestyle, tourism, demand, ageing effect, cohort effect, quantitative data 


\section{L'influence du vieillissement sur les pratiques touristiques en France}

\section{Introduction}

Si le vieillissement démographique est fréquemment évoqué comme porteur d'enjeux pour le secteur du tourisme dans les années à venir [Arnaud, Kovacshazy, 1998], c'est d'abord pour des raisons de croissance du marché potentiel, les retraités représentant déjà une clientèle très courtisée, tant à cause de sa disponibilité temporelle que du fait de son intérêt traditionnellement fort pour les voyages, et notamment pour les voyages organisés ou à forfait, intéressants pour les opérateurs. Mais cet intérêt s'explique aussi par le fait que les pratiques des retraités sont, sur de nombreux points, au cœur d'évolutions plus générales dans les façons de voyager. Pour apporter des éléments d'appréciation sur les changements en cours et sur la part prise par les « seniors » dans ces évolutions de comportements, nous présentons certains résultats d'un rapport réalisé pour le Conseil National du Tourisme ${ }^{1}$ sur le thème du tourisme à l'âge de la retraite [Pochet, Schéou, 2002].

Nous nous intéressons essentiellement aux retraités et préretraités, soit si l'on souhaite définir par l'âge cette catégorie et bien qu'il n'y ait pas de délimitation parfaite : les 60 ans et plus plutôt que les 50 ans et plus couramment distingués dans les études marketing, car c'est encore essentiellement le passage à la retraite qui définit les caractéristiques de ce grand groupe d'âge. Toutefois, pour des raisons de comparabilité avec des sources statistiques publiées, nous serons parfois amenés à présenter les comportements des 65 ans et plus et nous présenterons aussi ceux des 50-64 ans, pour essayer d'éclairer ce que pourront être les pratiques des jeunes retraités de demain.

Nous ferons principalement référence à des traitements statistiques que nous avons effectués à partir de l'enquête "Suivi de la Demande Touristique des Français » (SDT) de 1999 ${ }^{2}$, comparés, sur les mêmes classes d'âge avec celle de 1991 ou à défaut, avec celle de 1995, à partir de données déjà publiées [Blacodon, 1997]. Nous ferons aussi référence à certains résultats de l'Enquête Permanente sur les Conditions de Vie (EPCV) de $1999^{3}$. Ces deux enquêtes appréhendent des réalités différentes dans le sens où l'enquête SDT porte sur les déplacements touristiques ${ }^{4}$ (donnant lieu à un séjour d'au moins une nuitée hors du domicile quel que soit le motif ${ }^{5}$ ) et l'enquête Insee se limite aux voyages de vacances ou d'agrément d'au moins quatre nuits hors du domicile (ce qui exclut les courts séjours touristiques de moins de 4 nuits, les déplacements professionnels et les déplacements personnels motivés par des problèmes de santé, le décès d'un proche,...[Rouquette, 2000]). Autre différence : les taux de départ généraux publiés par la Direction du Tourisme à partir du SDT sont calculés en ramenant les touristes sur la population française âgée de 15 ans et plus ${ }^{6}$ alors que l'Insee ramène les vacanciers à la population totale pour calculer les taux de départ. Les deux sources d'information ne sont donc pas directement comparables mais complémentaires: les tendances de fonds qu'elles mettent en évidence sont assez convergentes.

\footnotetext{
${ }^{1}$ Organisme de conseil du Secrétariat d'Etat au Tourisme français.

${ }^{2}$ L'enquête SDT est une enquête mensuelle réalisée sur un panel de 20000 personnes dont la maîtrise d'ouvrage est détenue par la Direction du Tourisme.

${ }^{3}$ L'enquête EPCV est une enquête annuelle réalisée par l'Insee, qui comporte tous les 5 ans un volet important sur les vacances [Rouquette et Taché, 2002].

${ }^{4}$ Les définitions d'usage concernant les déplacements touristiques sont les suivantes : le voyage est l'unité de déplacement comprenant la période entre le départ du domicile et le retour au domicile, il doit comporter au moins une nuit hors du domicile, on parlera de court voyage pour des durées de une à 3 nuitées et de long voyage ou de vacances pour 4 nuitées et plus. Un voyage peut se décomposer en plusieurs séjours se définissant par le fait d'avoir passé au moins une nuit en un lieu fixe. La durée des voyages et des séjours s'exprime en nuitées.

${ }^{5}$ Nous avons pour notre part réduit le champ aux déplacements personnels (hors voyages à but professionnel).

${ }^{6}$ Par la suite, par souci de simplification, nous parlerons de l'ensemble de la population pour désigner la population âgée de 15 ans et plus pour toutes les statistiques issues du SDT.
} 


\section{Des évolutions socio-démographiques favorables au développement du tourisme à l'âge de la retraite}

\section{Les effets démographiques}

Le tourisme des retraités est porté par des évolutions démographiques et sociales structurelles fortes. Si l'on s'en tient à la répartition de la population par tranche d'âge, la France vieillit, à l'instar des autres pays industrialisés : les 60 ans et plus représentent $21,3 \%$ de la population contre $21,4 \%$ dans l'Europe des 15 ; dans les années 90, la population des 60 ans et plus s'est accrue de 1,2 millions de personnes, pour atteindre 12,5 millions en 1999. La participation croissante des personnes âgées au monde des vacances n'est donc pas une surprise. Or, le vieillissement démographique est encore très largement à venir dans nos sociétés, soulevant nombre d'interrogations tant sur le financement des systèmes de retraite, que sur la place des personnes très âgées dans notre société ou encore sur le niveau et la structure de la consommation. En effet, le nombre de personnes âgées va croître en part absolue (de 12,1 millions en l'an 2000 à 15,7 millions en 2015 soit $+30 \%$ ), et relative (de $20,6 \%$ à $25,3 \%$ de la population totale). Par le simple fait démographique, si les comportements demeurent inchangés, la demande émanant des 60 ans et plus croîtra donc de $+30 \%$ en volume. Comme dans le même temps, et toujours en raisonnant à comportements inchangés, le nombre absolu des plus jeunes va décroître légèrement, la part des plus de 60 ans dans l'ensemble des touristes et vacanciers ne peut que s'accroître fortement, notamment avec l'arrivée à la retraite des premières classes pleines du baby-boom à partir de 2006.

Au côté des évolutions purement démographiques, et de façon en partie liée à celles-ci, un certain nombre de faits de société transforment les modes de vie à la retraite. Le premier d'entre eux est l'élargissement de l'horizon de vie à la retraite. Si l'espérance de vie à la naissance a connu un gain de 6 ans en un quart de siècle pour atteindre 75,5 ans chez les hommes et 83 ans chez les femmes en 2001, c'est en grande partie du fait de gains de longévité particulièrement forts aux âges élevés. Aussi, l'espérance de vie à 60 ans est, en 2000 , de 20 ans chez les hommes et de 25,3 ans chez les femmes, contre respectivement 17,3 ans et 22,4 ans vingt ans plus tôt.

Deuxième fait important, qui concerne tout particulièrement la propension à voyager, l'allongement de l'espérance de vie est favorable à la prolongation de la vie en couple aux âges élevés. En effet les enquêtes le montrent, les personnes âgées hésitent beaucoup plus à partir lorsqu'elles vivent seules. Certes, alors que les situations de vie en couple demeurent très fréquentes chez les hommes (plus de $70 \%$ des cas entre 80 et 84 ans), dès l'âge de 75 ans, la vie de couple ne constitue plus la majorité des cas chez les femmes. Mais, chez les femmes aussi, la tendance actuelle est à un net accroissement des situations de vie maritale. En particulier, dans la tranche des 70-80 ans, on observe qu'en 12 ans (de 1984 à 1996), la proportion de femmes vivant en couple s'est accrue de plus de 10 points [Flipo et al. 1999].

\section{$\underline{\text { L'impact de l'état de santé }}$}

L'apparition plus tardive de la solitude est un facteur favorable au développement des départs en vacances à des âges de plus en plus élevés... à condition que l'état de santé reste suffisamment bon. Or, et c'est le troisième point qui nous semble important en la matière, les gains de longévité ne se sont pas faits au prix d'une dégradation de l'état de santé moyen : si les données très récentes manquent, il est à noter que dans les années 80, les gains d'espérance de vie sans incapacité (soit le nombre moyen d'années à vivre en bonne santé, une fois déduites les années vécues avec un handicap) ont été encore plus importants que les gains d'espérance de vie simple [Robine, 1994]. Il est généralement admis qu'en moyenne, à âge égal, on est plus apte à se déplacer et à mener une vie indépendante qu'il y a vingt ans. Néanmoins pour le moment, la croissance des taux de départ concerne moins les 70 ans et 
plus (passage de 32 à $38 \%$ en 20 ans), les effets du vieillissement redevenant progressivement plus prégnants. En effet, après 70 ans, les motifs de santé représentent le premier motif invoqué en cas de non-départ en vacances sur une année entière, d'après la dernière enquête EPCV de l'Insee (38\% des non-partants après 70 ans contre $15 \%$ chez les sexagénaires).

En ce qui concerne la santé, les effets futurs sur la demande touristique des personnes âgées restent incertains. Devraient agir positivement: l'amélioration de l'espérance de vie et notamment une durée moyenne de vie en couple un peu plus longue et, gains en matière de santé aidants, une période de vie plus importante en situation d'autonomie. Côté négatif: le vieillissement interne à la population âgée qui, en dépit des gains en matière de santé, pose la double question de l'accès aux vacances des personnes très âgées, et de l'aide familiale et de ses contraintes pour la génération des enfants, actifs en fin de carrière ou jeunes retraités (une personne âgée dépendante vivant à domicile sur deux ne reçoit d'aide que de sa famille). On peut par ailleurs penser que, parmi les futures générations de personnes très âgées, qui auront plus eu l'habitude de voyager que leurs devancières, le fait de ne plus pouvoir voyager sera vécu plus négativement qu'actuellement, créant ainsi de nouveaux besoins de vacances aux âges très élevés.

\section{$\underline{\text { L'impact du revenu }}$}

Les taux de départ sont fortement liés au revenu, en cela les données du SDT (figure 1) confirment la récente enquête Insee EPCV [Rouquette, 2001]. Même s'il est difficile d'isoler précisément l'influence de ce facteur, tant les handicaps sanitaires, sociaux (état de santé et degré d'isolement sont très variables selon le milieu social) et culturels sont étroitement liés aux limitations financières, les courbes rappellent la grande hétérogénéité de situations qui existe au sein de la population âgée.

\section{Figure 1 : Taux de partants en vacances pour différents groupes d'âge selon le revenu}

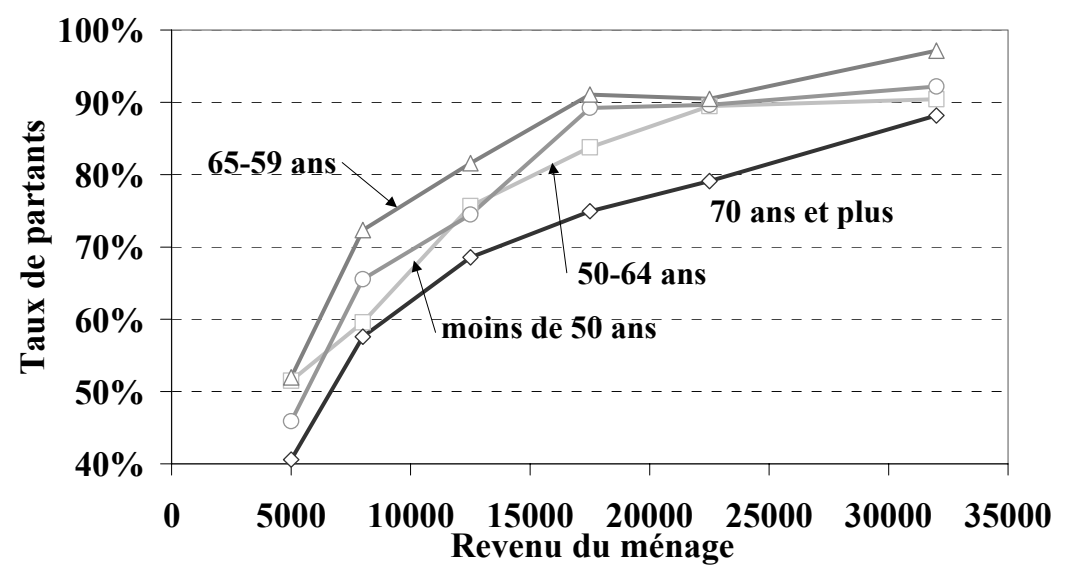

Source : Pochet et Schéou (2002) d'après le panel SDT de 1999

Les revenus se sont considérablement améliorés depuis trois décennies (revalorisation des montants des pensions et des minima sociaux, mais surtout, évolutions de nature générationnelle liées à la structure des professions et au développement du travail salarié féminin), et le niveau de vie moyen des retraités est désormais légèrement supérieur à celui des ménages d'actifs, même s'il faut souligner que de fortes inégalités de revenu traversent la population des retraités. De façon générale, les générations successives arrivant à l'âge de la retraite sont plus aisées que les précédentes. Cette tendance devrait continuer à se vérifier dans les prochaines années, avec l'arrivée progressive des générations nombreuses de l'aprèsguerre, même si les difficultés croissantes de financement des pensions de retraite, plutôt programmées à partir de 2010, devraient progressivement remettre en cause cet avantage relatif ce qui ne manquera pas d'avoir une incidence évidente sur les taux de partants, sur le 
nombre de départs dans l'année et sur les montants dépensés. Toutefois, la baisse prévisible des taux de remplacement pourra être compensée par des facteurs positifs comme la croissance des taux d'activité féminins, de telle sorte que si baisse de revenu des retraités il y a, elle devrait être d'ampleur très limitée d'ici à 10 ou 15 ans, comme le montrent des simulations basées sur la durée de carrière et les montants des salaires des générations successives [Bonnet et al., 1999]. Le recours plus fréquent à l'épargne individuelle pour compléter les pensions de retraite risque cependant d'accroître à l'avenir les inégalités de niveau de vie parmi les retraités. Enfin, en lien avec l'évolution des revenus, l'âge moyen de départ à la retraite est lui aussi à considérer. Compte tenu des réformes en cours et probables, il devrait remonter, mais lentement, de deux ans en une quinzaine d'années. Cette évolution jouera sans doute plus sur la disponibilité temporelle que sur le niveau de consommation touristique.

\section{Le rôle de la taille de l'agglomération de résidence}

La santé et le revenu ne sont pas les seuls facteurs influençant les taux de départ, la taille de l'agglomération et la région de résidence jouent aussi un rôle, même si leur impact apparaît plus limité (tableau 1). En zones rurales, les retraités comme les plus jeunes partent moins en vacances et le taux de départ va croissant avec la taille de l'agglomération. Une partie de ces différences de pratiques selon le lieu de vie est liée aux écarts de revenu existant entre les différentes zones. Mais les facteurs socioculturels, et la moins grande habitude de voyager de certaines professions, liée notamment à une plus faible disponibilité temporelle (agriculteurs, travailleurs indépendants), auxquels il faut ajouter l'influence propre du contexte spatial, continuent à jouer après 60 ans.

Tableau 1 : Taux de départ et agglomération de résidence en 1991 et en 1999

\begin{tabular}{|l|cc|cc|}
\hline & \multicolumn{2}{|c|}{1991} & \multicolumn{2}{c|}{1999} \\
\cline { 2 - 5 } & Moins de 65 ans & 65 ans et + & Moins de 65 ans & 65 ans et plus \\
\hline Commune rurale & $69,2 \%$ & $67,5 \%$ & $68,0 \%$ & $59,9 \%$ \\
$2000-20000$ habitants & $75,4 \%$ & $72,1 \%$ & $67,7 \%$ & $65,8 \%$ \\
$20000-100000$ habitants & $77,4 \%$ & $79,4 \%$ & $72,7 \%$ & $72,9 \%$ \\
100000 habitants et plus & $80,4 \%$ & $78,5 \%$ & $79,6 \%$ & $74,6 \%$ \\
Agglomération parisienne & $91,0 \%$ & $88,2 \%$ & $89,4 \%$ & $84,6 \%$ \\
\hline Ensemble & $77,4 \%$ & $76,9 \%$ & $74,5 \%$ & $71,0 \%$ \\
\hline
\end{tabular}

Source : panels SDT, Blacodon (1997) pour 1991 et Pochet, Schéou (2002) pour 1999.

Ces écarts entre villes et campagnes ne tendent pas à se résorber dans les années récentes. En revanche, à travers l'analyse des taux de départ par région de résidence, la spécificité francilienne ressort, mais concernant les autres régions, on constate un resserrement des taux de départ chez les plus de 65 ans entre 1995 et 1999, autour de 65 à 70\%, avec notamment une légère amélioration de la situation relative des retraités du nord de la France.

La conjonction de facteurs favorables aux déplacements touristiques des retraités permet de comprendre l'évolution positive des taux de partants que connaît cette catégorie de population depuis deux décennies, même si les retraités sont toujours un peu moins nombreux à partir en vacances que les plus jeunes. Ainsi, d'après le SDT, on constate une croissance de $10 \%$ du nombre de séjours et de $8 \%$ du nombre de nuitées de 1995 à 1999 chez les 65 ans et plus. Audelà des fluctuations de court terme, ces évolutions récentes reflètent une tendance de fond de nature générationnelle, comme le montre la comparaison des parcours des générations sur trente ans à partir des enquêtes Insee [Rouquette, 2000]. La cohorte née dans les années 30, âgée de 60 à 69 ans en 1999, compte ainsi 58\% de partants dans ses rangs à cette date, alors que la cohorte née dans les années 20 n'en comptait que 52\% lorsque, 10 ans plus tôt, elle est arrivée aux mêmes âges et la cohorte née dans les années 1910-1920, seulement 45\% encore dix ans plus tôt (figure 2). 

(séjours personnels d'au moins 4 nuits)

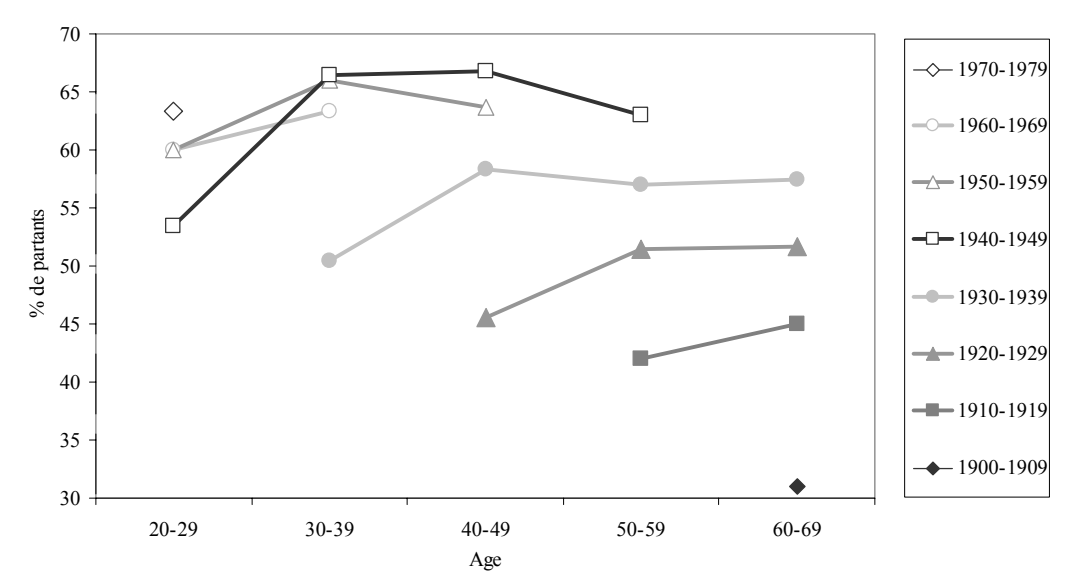

Lecture : chaque segment représente l'évolution des comportements moyens d'une génération selon son âge (la génération 1900-1909 correspond à ceux qui sont nés entre 1900 et 1909.

Source: enquêtes Insee EPCV 1999 et ECM de 1969, 1979 et 1989 [Rouquette, 2001].

Le changement est spectaculaire, puisqu'en deux décennies, c'est un sexagénaire sur quatre de plus qui part en vacances! A cela il faut ajouter un nombre moyen de séjour par partant qui est en croissance forte dans les années 90 chez les plus de 55 ans, avec des maxima de 2,6 à 2,4 séjours de vacances par an et par an enregistrés entre 55 et 70 ans (figure 3).

Figure 3 : Evolution du nombre moyen de séjours de 4 nuits et plus par partant entre 1989, 1994 et 1999 (enquête EPCV de l'Insee)

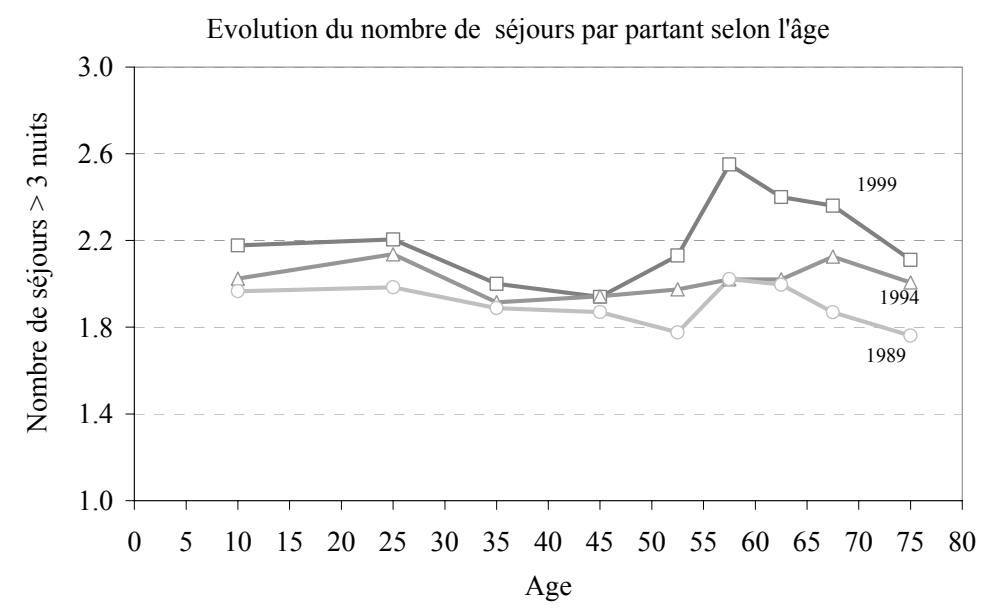

Lecture: Chaque classe d'âge est représentée par son centre de classe (exemple : les 0-19 ans sont affectés à la valeur 10)

Source : d'après les résultats des Enquêtes Insee EPCV de 1999 et ECM de 1989 et 1994. [Rouquette, Taché, 2002].

\section{Les pratiques touristiques à l'âge de la retraite}

\section{Les motifs de séjours : visites ou séjours d'agrément}

Toutes durées confondues, deux grands types d'activité motivent les séjours personnels des Français : les séjours d'agrément (vacances, tourisme, loisirs) et les visites aux parents et amis (figure 4). La durée moyenne des séjours pour motifs d'agrément est supérieure à celle des visites à la famille et aux amis et cette différence de durée entre motifs augmente avec l'âge, passant de 3,4 jours chez les moins de 50 ans à 4,8 jours chez les 70 ans et plus.

En termes d'évolution, le recul temporel manque car nous ne disposons ici que des données de 1995 et de 1999. Chez les plus de 65 ans, sur ces quatre années, la tendance est à la baisse du nombre de séjours motivés par des visites à la famille ou aux amis, au profit des séjours d'agrément, de santé ou autres, et ce plus encore chez les retraités que chez les plus jeunes. Cette évolution affectant les seniors est cependant moins nette en nombre de nuitées, en raison d'un raccourcissement de leur durée moyenne de séjour pour motif d'agrément (de 10,1 à 9,9 nuits) et d'un allongement de la durée moyenne de leurs séjours pour visites (de 5,7 
à 5,8 nuits). Enfin, cette tendance plus favorable au séjour d'agrément pourrait bien se prolonger dans les années à venir, car elle est actuellement visible aussi chez les 50-64 ans, qui tendent de plus à fractionner leurs départs et à réduire leur durée de séjour, tant d'agrément (de 8,6 à 8,3 nuits) que de visites (de 4,7 à 4,2 nuits).

Figure 4 : Répartition des séjours personnels en 1999 selon l’âge et le motif (\%)

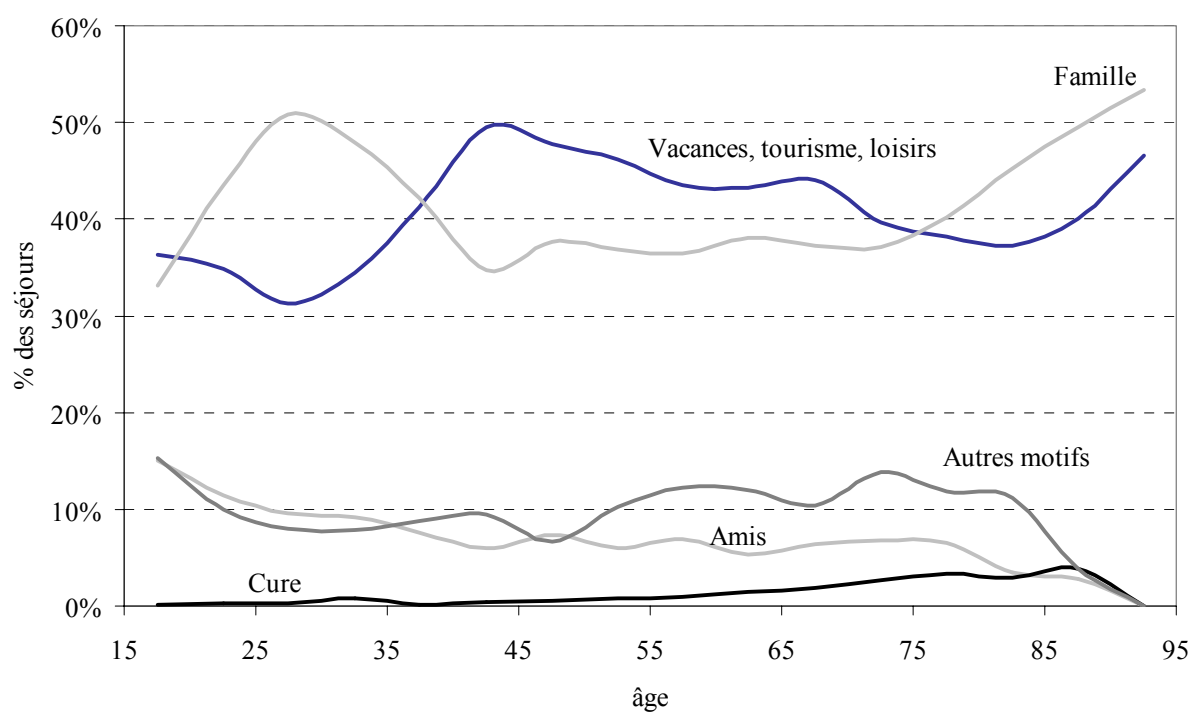

Source : Pochet et Schéou (2002) d'après le panel SDT de 1999

Les modes d'hébergement : l'importance du non-marchand et des résidences secondaires

Bien qu'en léger retrait au cours des années 90, l'hébergement non marchand (chez des parents ou amis ou dans une résidence secondaire) représente toujours, de loin, le premier mode d'hébergement des touristes français avec près de $60 \%$ de l'ensemble des nuitées (tableau 2). La prédominance de l'hébergement non marchand apparaît encore un peu plus marquée aux âges élevés : $62 \%$ des nuitées chez les plus de 65 ans, entre 55 et $60 \%$ du total des longs séjours à partir de 55 ans et $53 \%$ avant cet âge. En particulier, la ressource procurée par les résidences secondaires comme mode d'organisation des vacances est importante (près d'un séjour sur cinq). La possession de résidence secondaire est surtout le fait de ménages de plus de 50 ans, aussi n'est-il pas étonnant que ce mode d'hébergement tienne une place déterminante chez les retraités. En proportion de l'ensemble des nuitées, cette part monte à plus de 30\%. Son importance croît même ces dernières années (ce que confirment aussi les enquêtes Insee), de près de 5\% sur l'ensemble des nuitées des 65 ans et plus entre 1991 et 1999.

Tableau 2 : Evolution du mode d'hébergement pour différents groupes d'âge, en \% du total des nuitées personnelles, données des panels SDT de 1991 et 1999

\begin{tabular}{|l|cccc|cc|}
\cline { 2 - 7 } \multicolumn{1}{c|}{} & \multicolumn{2}{c}{ Moins de 65 ans } & \multicolumn{2}{c|}{ dont 50-64 ans } & \multicolumn{2}{c|}{65 ans et plus } \\
\cline { 2 - 7 } \multicolumn{1}{c|}{ Hébergement non marchand } & 1991 & 1999 & 1991 & 1999 & 1991 & 1999 \\
Dont : Famille/Amis & 58,0 & 55,5 & 62,7 & 57,8 & 63,7 & 62,2 \\
Dont : Résidence Secondaire & 42,6 & 41,6 & 32,9 & 30,1 & 37,8 & 31,7 \\
Hébergement marchand & 15,5 & 13,9 & 29,7 & 27,7 & 25,9 & 30,5 \\
Dont : Hôtel & 42,0 & 44,5 & 37,3 & 42,2 & 36,3 & 37,8 \\
Dont : Location & 8,6 & 11,4 & 13,0 & 14,2 & 15,4 & 14,9 \\
\hline Total & 10,2 & 10,0 & 8,5 & 8,9 & 10,2 & 6,9 \\
\hline
\end{tabular}

Source : Panels SDT, Blacodon (1997) pour 1991 et Pochet et Schéou (2002) pour 1999. 
Au-delà du facteur-coût bien évidemment déterminant, l'importance du "non-marchand" reflète aussi des pratiques de vacances spécifiques, comportements vis-à-vis desquels le secteur de l'hébergement touristique a du mal à proposer des formules adaptées. La résidence secondaire comme l'hébergement chez des proches permettent dans bien des cas de rassembler des groupes familiaux (les différentes générations d'une même famille, par exemple) et amicaux, ce qui est plus difficile avec l'hôtel ou la location. Ces évolutions peuvent donc s'interpréter comme le résultat du rôle important du réseau amical et affectif dans l'organisation des vacances, mais aussi comme la conséquence de l'expérience croissante des Français (et notamment des plus âgés) qui peuvent se passer des circuits marchands pour une part croissante de leurs séjours.

Avec le recours fréquent à la résidence secondaire, des pratiques de double résidence, ou de résidence alternée apparaissent. Les jeunes retraités, ne voulant pas se retrouver confinés dans « des régions à seniors », éviteraient de déménager tout en compensant cette absence de migration de retraite par des migrations saisonnières [Dieudonné, 1997]. Ce nouveau modèle résidentiel demeure encore très minoritaire, ne serait-ce que du fait de la diffusion malgré tout assez limitée des résidences secondaires mais il pourrait se développer un peu à l'avenir. De plus, les double-résidences ont un impact sur la demande touristique qui dépasse largement leur importance numérique limitée, ne serait-ce que par la multiplication des voyages qu'elles peuvent entraîner au cours de l'année.

\section{La saisonnalité : un rapport au temps spécifique à la retraite}

Pour les adultes en âge d'activité, comme pour les enfants scolarisés et les étudiants, le temps des vacances constitue un temps non contraint ou moins contraint (où la volonté de se reposer, de se détendre l'emportent) que le temps quotidien. En revanche, pour les retraités, les vacances constituent un temps organisé, investi, un temps plein, que l'on souhaite "chargé d'activités pour s'évader du vide » créé par la cessation de l'activité professionnelle [Clary, 1993]. Certes, les périodes de vacances et les voyages ne sont pas les seules activités qui permettent de fournir des points de repère dans l'année, de structurer le temps à la retraite : activités associatives voire bénévoles ou para-productives et de sociabilité sont de plus en plus partagées. Il n'en demeure pas moins que les temps de vacances ne sont pas affectés de la même signification à la retraite que pendant la vie active.

Paradoxalement, ces significations différentes des temps de vacances à la retraite et pendant la période d'activité permettent de comprendre pourquoi la saisonnalité des départs des seniors n'est pas si différente que cela de celle, fortement marquée, des actifs. Les vacances doivent être aussi un temps de rencontre et de rapprochement des plus jeunes générations, aussi le taux de départ sur les trois mois d'été s'établit à $51 \%$ chez les 65 ans et plus contre $57 \%$ en moyenne. Si les retraités souhaitent partir l'été comme les autres vacanciers, c'est sans doute en partie pour profiter des beaux jours, et aussi pour ne pas bouleverser leurs habitudes antérieures. Mais c'est aussi dans bien des cas pour ne pas avoir à se retrouver dans un quartier déserté l'été, ou dans un village de vacances aux trois quarts vide en basse saison ou occupé uniquement par d'autres retraités, c'est enfin pour pouvoir se retrouver avec des parents proches, qui eux ont des contraintes temporelles. Ce désir de rapprochement a des limites : la différenciation tarifaire selon la haute, la moyenne et la basse saison, stratégie courante de la part des opérateurs pour mieux répartir leur activité, entraîne naturellement une certaine concentration des personnes âgées sur les périodes creuses : moins contraintes sur le plan professionnel et familial, elles s'orientent logiquement vers les dates les moins chères.

Cet étalement des séjours dans l'année constitue bien une caractéristique structurelle de la demande touristique des personnes âgées, et ce depuis nombre d'années. Les différentes sources convergent, même si les panels SDT mettent en évidence une différenciation moins marquée que l'enquête EPCV. En effet, les courts séjours personnels, recueillis dans le panel SDT mais pas dans l'enquête Insee sont mieux répartis dans l'année que les longs séjours, 
chez les actifs. Aussi, par rapport aux personnes actives, les taux de départs mensuels des retraités ne sont réellement nettement plus faibles qu'au mois d'août (figure 5). Il n'en demeure pas moins vrai qu'en structure, le déséquilibre n'est pas négligeable pour ce mois (19,3\% soit près d'un séjour sur cinq avant 60 ans, contre $11,1 \%$ après cet âge), mais aussi en juin, septembre et octobre $(30,5 \%$ du total pour ces trois mois après 60 ans, contre $17,7 \%$ avant cet âge). Les autres mois de l'année offrent une répartition nettement plus équilibrée entre actifs et retraités, notamment le mois de juillet (12,3\% avant 60 ans, 9,8\% après 60 ans).

Figure 5 : Saisonnalité des séjours personnels, toutes durées confondues selon la date de départ en 1999

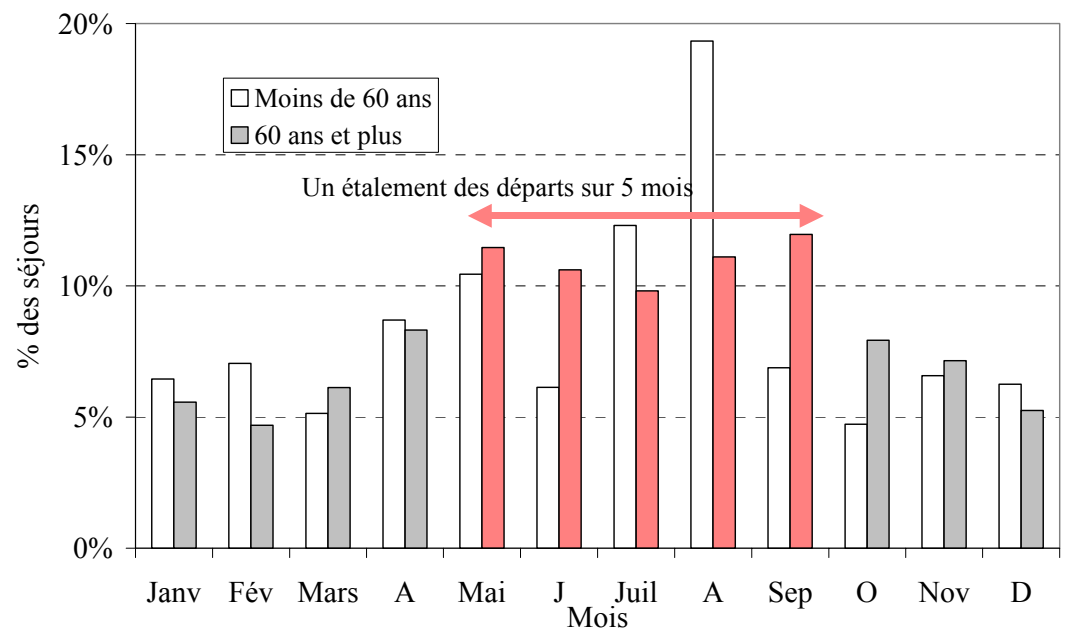

Source : Pochet et Schéou (2002) d'après le panel SDT de 1999

Les espaces de villégiature : un attrait pour le tourisme vert... et pour le tourisme urbain

La part des nuitées passées en dehors du domicile par les plus âgés est relativement plus favorable aux zones rurales, et moins aux lieux de villégiature situés sur le littoral (figure 6) qui voient leur attraction diminuer dès la tranche d'âge 40-50 ans. A partir de cette même tranche d'âge, les lieux de destination urbains voient leur fréquentation augmenter avant de diminuer après 70 ans.

Figure 6 : Répartition des nuitées entre les espaces de destination selon l’âge (\%)

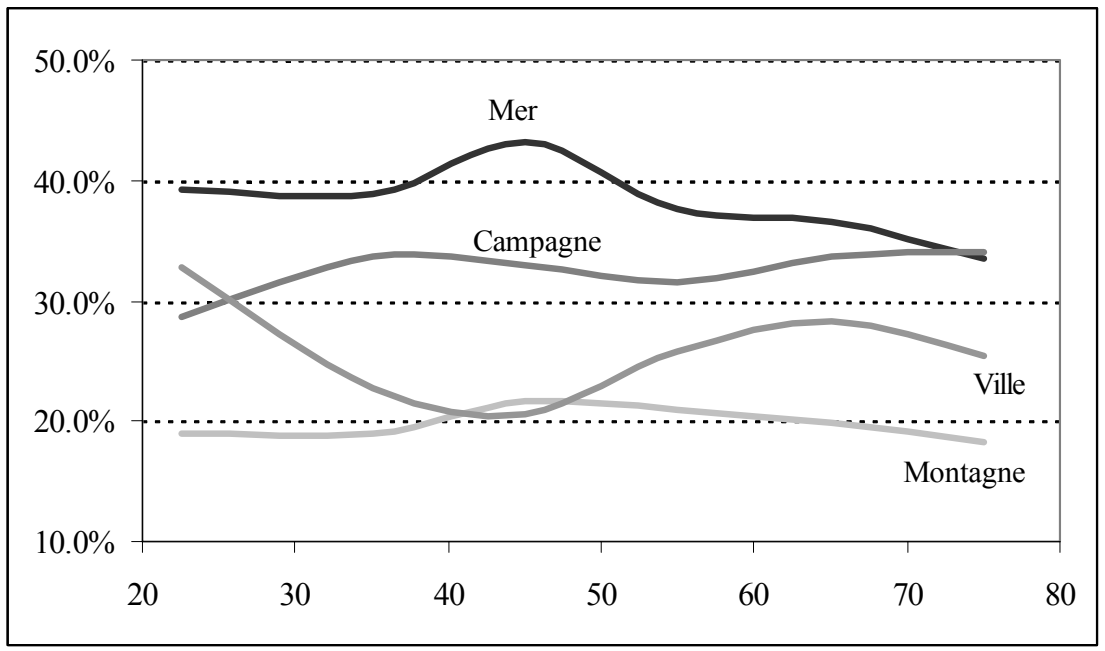

Lecture : près de $40 \%$ des nuitées personnelles des moins de 35 ans sont passées à la mer (le total peut dépasser $100 \%$, plusieurs espaces différents pouvant être fréquentés lors d'un séjour).

Source : Pochet et Schéou (2002) d'après le panel SDT de 1999

Les données Insee de 1999 confirment ces résultats, dans le cas des séjours de vacances de 4 jours et plus uniquement. L'intérêt pour les espaces urbains est particulièrement marqué chez les plus de 60 ans et se réduit après 70 ans, et s'explique d'une part par le développement du 
tourisme culturel, mais aussi par l'importance du motif « visites aux parents » (parents qui résident bien souvent en milieu urbain). Concernant la montagne, c'est le moindre attrait, assez logique, pour les sports d'hiver qui explique le relatif tassement observé chez les plus de 55 ans pour ce type de destination car les seniors sont proportionnellement aussi, sinon plus, nombreux que les plus jeunes à pratiquer les autres types de séjours à la montagne.

Enfin, l'attrait pour la campagne, plus prononcé dès 55 ans, ne s'explique pas seulement par un effet d'âge (préférence pour le calme et le repos), car cet engouement pour le « tourisme vert » va croissant dans les années 90 , et ce en particulier chez les 65 ans et plus. Il semble que les générations successives de retraités sont de plus en plus sensibles aux richesses (patrimoine, terroir, cultures locales, gastronomie) des régions françaises.

\section{Les modes de transport : la voiture d'abord}

Reflet de voyages internes à la France métropolitaine à plus de $80 \%$, le mode de transport privilégié est la voiture particulière, quelle que soit la classe d'âge : 75 à $80 \%$ pendant la vie active, et encore près de 70\% ensuite sur l'ensemble des séjours (tableau 3), et même plus de $70 \%$ chez les sexagénaires (d'après l'enquête EPCV de l'Insee de 1999, sur les longs séjours d'été).

Au cours des années 90, c'est le mode aérien qui connaît la croissance la plus forte dans toutes les tranches d'âge. Prendre l'avion ne fait plus peur aux retraités qui utilisent tout autant ce mode que les plus jeunes. Mais son usage est encore très minoritaire, car demeurant à tous âges essentiellement réservé aux voyages d'agrément réalisés à l'étranger.

Tableau 3 : Evolution dans l'usage des modes de transport de 1991 à 1999 en \% de l'ensemble des séjours personnels

\begin{tabular}{|l|cccc|cc|}
\hline \multirow{2}{*}{} & \multicolumn{2}{|c}{ Moins de 65 ans } & \multicolumn{2}{c|}{ Dont 50-64 ans } & \multicolumn{2}{c|}{65 ans et plus } \\
\cline { 2 - 7 } & 1991 & 1999 & 1991 & 1999 & 1991 & 1999 \\
\hline Voiture & 76,0 & 77,5 & 75,2 & 74,9 & 66,4 & 68,1 \\
Train & 12,8 & 10,6 & 13,1 & 10,0 & 20,6 & 16,6 \\
Avion & 5,5 & 7,0 & 5,9 & 9,0 & 5,5 & 7,3 \\
Autocar & 2,4 & 2,1 & 3,6 & 3,2 & 6,1 & 5,7 \\
Autre & 3,3 & 2,7 & 2,1 & 2,8 & 1,4 & 2,3 \\
\hline Total & 100,0 & 100,0 & 100,0 & 100,0 & 100,0 & 100,0 \\
\hline
\end{tabular}

Source : Panels SDT, Blacodon (1997) pour 1991 et Pochet et Schéou (2002) pour 1999.

Chaque génération arrivant à la retraite est un peu plus motorisée que la précédente [Bodier, 1996], et ces effets de génération sur la motorisation des ménages retraités ont un net impact dans la période récente. Selon les panels SDT, l'automobile gagne 1,7\% chez les plus de 65 ans, sur l'ensemble des séjours, ce que confirment les enquêtes Insee sur les séjours de vacances d'été. Par rapport à 1993, si la part de marché de la voiture particulière se rétracte chez les moins de 55 ans (-1,8 points en moyenne sur l'ensemble de la population), elle a gagné 5\% entre 60 et 69 ans (elle se situait à $66 \%$ chez les sexagénaires en 1993) et 3\% chez les plus de 70 ans.

Cette évolution se fait essentiellement au détriment du train et du car, modes de transport en concurrence directe avec la voiture. L'autocar occupe une place marginale dans l'ensemble des séjours d'été $(3,5 \%)$, mais demeure plus utilisé par les seniors $(5 \%$ environ chez les sexagénaires) que chez les adultes en âge d'activité. Mais l'information la plus importante est le fait que l'autocar est de plus en plus utilisé par les plus de 70 ans : $13 \%$ en 1999 contre 9\% en 1993. Cette évolution est récente. En 1993, les 60 ans et plus l'utilisaient beaucoup plus uniformément ( 8 à $9 \%$ au cours des séjours d'été selon l'Insee [Monteiro 1996]. Cette concentration de l'usage de l'autocar chez les plus de 70 ans lui permet de maintenir sa part 
de marché sur les séjours d'été de plus de 4 jours (3,3\%) mais rend sa position fragile : les futurs septuagénaires, plus motorisés et moins adeptes des voyages en groupe préconstitué, risquent fort de se tourner vers la voiture ou vers d'autres modes de transport. L'« automobilité » de plus en plus répandue après 60 ans tend à rapprocher les citadins âgés des plus jeunes en matière de mobilité locale, avec l'élargissement des espaces de vie quotidiens [Pochet, 1997]. De la même façon, elle est sans aucun doute aussi un facteur d'autonomie favorable au développement des voyages personnels chez les retraités, et notamment des "courts séjours" et des déplacements de loisirs, tout au moins tant que la forme physique permet encore d'envisager des voyages qui peuvent s'avérer éprouvants.

Tels qu'ils sont appréhendés par ces enquêtes quantitatives, pendant les vacances les comportements moyens des retraités ne diffèrent pas fondamentalement de ceux des actifs, les évolutions récentes étant à un rapprochement de leurs pratiques de celles des plus jeunes. Qu'il s'agisse de la durée de séjour, en diminution (même si les personnes âgées demeurent toujours un peu moins adeptes du court séjour, cela pourrait évoluer), de l'individualisation croissante des modes d'organisation (par l'usage préférentiel des modes de transport privés, la voiture particulière, sur les modes collectifs), du recours moins fréquent aux formules « tout compris ", de la prédominance à peine entamée de l'hébergement non marchand à tous les âges, ou encore des espaces fréquentés assez proches,... assez peu de choses les séparent des actifs en vacances. Certes, l'appréhension quantitative des phénomènes touristiques n'est pas aisée, tant la frontière avec les autres pratiques de loisirs est floue et mouvante [Viard, 2001] et les statistiques, même précises, tendent à réduire malgré tout les pratiques touristiques à leur enveloppe, les contenus pouvant différer plus fortement selon l'âge.

\section{Conclusion}

Pour conclure, il nous semble tout d'abord important de rappeler que le principal déterminant des pratiques touristiques des retraités n'est sans doute pas le revenu stricto sensu, mais est plus sûrement à rechercher du côté de la génération d'appartenance et des facteurs culturels (et notamment les pratiques développées à de plus jeunes âges) spécifiques à cette dernière, puis du côté de l'âge, du fait de son caractère progressivement ou brutalement contraignant sur la mobilité.

L'impact durable des expériences et des habitudes acquises au cours des plus jeunes années sur les niveaux et les formes de consommation à la retraite, incitent à penser que la demande touristique émanant des retraités devrait connaître de nouvelles inflexions dans les prochaines années, avec l'arrivée des générations nées après-guerre. Ces cohortes, qui ont été au cœur des «trente glorieuses » et ont traversé les années de crise sans trop d'encombre ont, de façon générale une situation sociale et un niveau de vie moyen nettement plus élevés que les précédentes, et vraisemblablement, que les suivantes [Chauvel, 1998]. Ayant pu prolonger leurs études en moyenne plus longtemps, elles ont connu aussi dès leur jeune âge l'émancipation féminine à travers le développement du travail salarié, les années de forte consommation, le développement des loisirs, des échanges, des médias et des voyages. Ces générations tranchent nettement avec celles nées pendant la première moitié du siècle, notamment dans le domaine des pratiques de loisirs. Le grand paradoxe de ces générations, que l'on retrouve dans leurs comportements ultérieurs, est d'avoir été au cœur de la critique de la société de consommation, tout en étant les premières à se distinguer autant de leurs devancières par le développement de la consommation, mais aussi par la diversification, "l'individualisation" des produits et des services consommés [Préel, 2000].

Des changements de comportements touristiques à la retraite sont donc à attendre des babyboomers, dont une grande partie a une expérience importante du monde des voyages même si, il faut le rappeler, comme toute génération, elle n'est pas homogène du point de vue de sa 
composition sociale. On peut poser comme hypothèse qu'une fois libérés des contraintes professionnelles et familiales, la majorité des membres de cette génération sera animée d'un fort désir de voyages. Certes, ces générations compteront toujours une proportion de personnes ne se déplaçant pas ou peu, soit par choix soit par contrainte. Mais avec le renouvellement des générations, le taux de partants devrait logiquement s'accroître encore un peu chez les plus de 60 ans. Parmi les partants, la variété des pratiques sera sans doute de plus en plus grande, car les différences culturelles n'ont pas tendance à se réduire. De façon globale, l'arrivée des générations nées après-guerre à la retraite ne se traduira sans doute pas réellement par une rupture, mais plutôt par une inflexion et une accélération progressives de tendances déjà à l'œuvre comme l'individualisation des pratiques, le raccourcissement des séjours et l'enchaînement de séjours de nature différente au cours de l'année chez les partants. On pourrait assister ainsi à un développement des pratiques consommatoires et d'aubaine, à un développement des destinations lointaines et " originales », des séjours culturels ou liés à une activité bénévole ou fortement revendiquée ou structurante, coexistant avec des pratiques plus classiques et des séjours plus sédentaires, à l'image de celles qui sont dominantes actuellement.

\section{Références}

ARNAUD G. et KovaCSHAZY C. (rapporteurs), ViaRD J. (président) (1998), Réinventer les vacances, la nouvelle galaxie du tourisme. Premier Ministre, Conseil Général du Plan, Secrétariat d'Etat au Tourisme, Paris, La Documentation Française.

BLACODON M. (1997), Le tourisme des seniors 1991/1995, Paris, Observatoire national du tourisme, coll. "Les essentiels".

BoDier M. (1996), "La voiture, une habitude qui se prend jeune”, Insee Première, nº 474.

Bonnet C., Chanut J.-M., et al. (1999), "Des retraites qui vont continuer à croître", INSEE, Données Sociales 1999, pp. 427-432.

Chauvel L. (1998), Le destin des générations, Paris, PUF.

ClARY D. (1993), Le tourisme dans l'espace français, Paris, Masson.

DiEudonné E. (1997), “Les seniors : un monde éclaté !” Cahiers Espaces, n 54, pp. 20-22.

FLIPO A., LE BLANC D. et LAFERRÈRE A. (1999), "De l'histoire individuelle à la structure des ménages", Insee Première, $\mathrm{n}^{\circ} 649$.

MONTEIRO I. (1996), Les vacances des Français. Tendances longues et résultats détaillés de 1993 à 1994, INSEE, coll. Insee Résultats n 451-452.

POCHET P. (1997), Les personnes âgées, Paris, La Découverte, coll. « Repères ».

POCHET P. et SchÉOU B. (rapporteurs) SOUlAGE F. (prés.) (2002), Le tourisme à l'âge de la retraite, Secrétariat d'Etat au Tourisme, Paris, La Documentation Française.

PrÉEL B. (2000). Le choc des générations, Paris, La Découverte.

RoBINE, A. (1994). "L'évolution et l'espérance de vie sans incapacité à 65 ans." Gérontologie et société $\mathrm{n}^{\circ} 71$.

RouQueTtE C. (2000), "Chaque année quatre français sur dix ne partent pas en vacances", Insee Première $\mathrm{n}^{\circ} 734$.

RouQueTte C. (2001), "Départs en vacances : la persistance des inégalités", Economie et Statistique, $\mathrm{n}^{\circ} 345$, pp. 33-53.

ROUQUETTE C et TACHÉ C. (2002), “Les vacances des français“, Insee Résultats n4, mai 2002.

VIARD J. (2001), "Temps libres: le triangle des Bermudes de l'aménagement du territoire", Territoires 2020 Revue d'Etudes prospectives de la DATAR, $\mathrm{n}^{\circ}$ 3, pp. 55-60. 